\title{
CORRELATION OF THE MURMUR OF INTERVENTRICULAR SEPTAL DEFECT WITH PRESSURE DIFFERENCES BETWEEN THE VENTRICLES*
}

\author{
BY
SIDNEY FENIG, JOSEPH HILSENRATH, LEONARD STEINFELD, JERRY LASSER, GABRIEL GENKINS, AND ALVIN J. GORDON \\ From the Division of Cardiology, Department of Medicine, and the Department of Pediatrics, The Mount Sinai Hospital, \\ New York, N.Y., U.S.A. \\ Received May 1, 1964
}

The systolic murmur associated with interventricular septal defect presents a number of variations which have been related to the location and size of the defect, the pulmonary vascular resistance, and to the pressure gradient between the ventricles. The purpose of this investigation was to study the relation of the pressure gradient in interventricular septal defect to the time-intensity characteristics of the murmur. This was accomplished by simultaneously recording pressure pulses in both ventricles together with continuous pressure differences between the ventricles, and phonocardiograms.

\section{SUBJECTS AND METHODS}

Twenty patients with isolated interventricular septal defect were studied. Left-to-right shunts of varying size and location with and without pulmonary hypertension were encountered. The diagnoses were supported by right heart catheterization and by left ventricular angiocardiography, and in one patient (L.R.) by operation. Ages ranged from $1 \frac{1}{2}$ to 18 years. The following parameters were recorded simultaneously and with no significant parallax error on an Electronics for Medicine oscilloscopic recorder: equisensitive right and left ventricular pressure pulses and continuous pressure differences between the two ventricles by electronic subtraction; lead II of the electrocardiogram; phonocardiograms recorded from the point on the chest where the murmur was loudest, usually with both high and low frequency filtration.

Right heart catheterization was performed by standard techniques. Left heart catheterization was done by arteriotomy of either the brachial or femoral artery (usually the former) and retrograde passage of a specially designed NIH catheter with a J-shaped tip, size $6 \mathrm{~F}$ or 7F, into the left ventricle. Angiocardiograms from the left ventricle were obtained in all patients at the end of the procedure and established the diagnosis of interventricular septal defect as well as the location of the defect in each case. The classification of Baron et al. (1963) was used in defining the anatomy of the lesions (Fig. 1).

Pressures were recorded by means of Statham P23G transducers. The microphone was of a ceramic crystal displacement type with flat frequency response from 30 to 1000 cycles per second (Electronics for Medicine). By means of branched plugs the output was fed through two separate pass band filters in the ranges of 25 to $250 \mathrm{cps}$ (low frequency) and $250-2500 \mathrm{cps}$ (high frequency). The murmur was usually best recorded in the high frequency range. Recordings were made at both 50 and $150 \mathrm{~mm}$./sec. paper speed with time lines at 0.04 and $0.02 \mathrm{sec}$. The zero point for pressure was at the mid-thoracic level.

Morphine and scopolamine premedication was given, and in the younger patients this was supplemented by intravenous or rectal pentothal sodium. One patient with a muscular defect was given phenylephrine intravenously to observe the effect on the murmur and on the pressure difference curve.

* This investigation was supported, in part, by a research grant $(\mathrm{H}-2168)$ from the National Heart Institute, U.S. Public Health Service. 


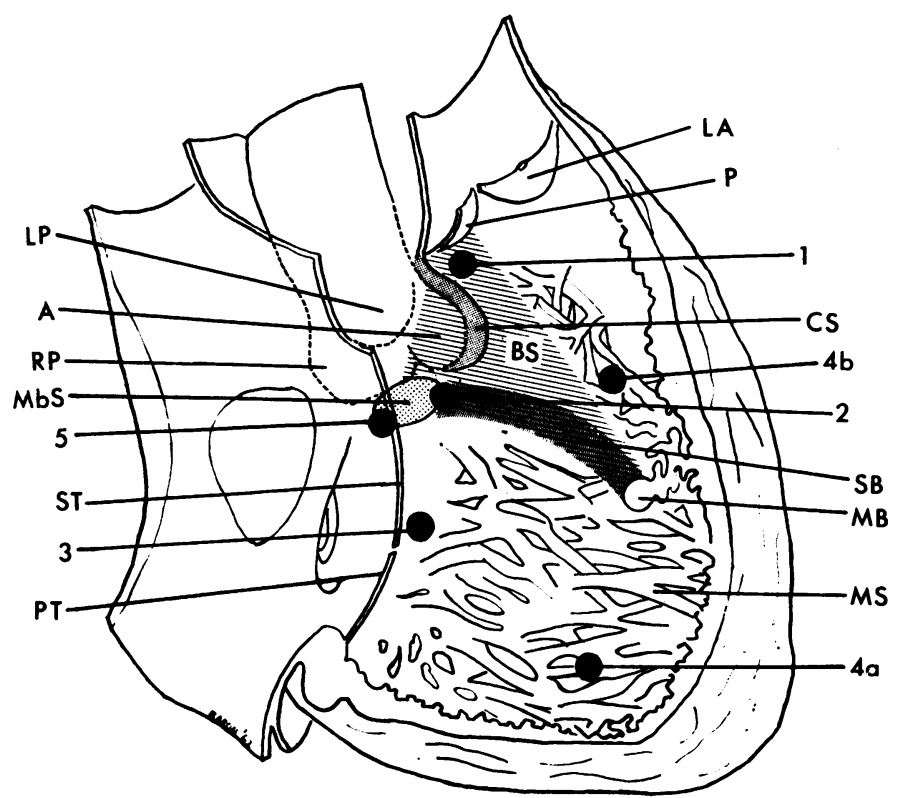

FIG. 1.-Diagrammatic representation of the right ventricular aspect of the ventricular septum. The location of the aortic root is indicated by the dotted lines. The septum is divided into its bulbar (BS), membranous (Mbs), and muscular (MS) portions. The black circles indicate the locations of the different types of ventricular septal defects: Type 1 , bulbar septal defect; 2 , membranous ventricular septal defect; 3 , posterior muscular septal defect; $4 \mathrm{a}$, low anterior muscular septal defect; 4b, high anterior muscular septal defect; 5 , membranous atrio-ventricular septal defect. LP, left posterior aortic leaflet; RP, right posterior aortic leaflet; $A$, anterior aortic leaflet; LA, left anterior pulmonic leaflet; $P$, posterior pulmonic leaflet; SB, septal band; ST, septal leaflet of the tricuspid valve; PT, posterior leaflet of the tricuspid valve. (Reprinted, with permission of the publisher, The Radiological Society of North America, from Radiology (1963), 81, 223.)

\section{RESULTS}

The pressure difference curves in patients without pulmonary hypertension characteristically conformed to the left ventricular pressure pulses in shape, being roughly rectangular. In patients with pulmonary hypertension the shape of the pressure difference curve depended on the height of the right ventricular pressure. When the right ventricular pressure was only moderately raised, the pressure difference curve was rectangular as described above. In the presence of marked right ventricular hypertension, however, there were slight differences in pressure between the two ventricles which varied from one case to another without any set pattern. In addition, the pressure artefacts in these cases were of an order of magnitude that nullified the slight pressure differences encountered.

As a general rule the murmurs varied much from case to case and only occasionally conformed to the pressure difference curve. In addition, there was little or no correlation from one patient to another between the over-all intensity of the murmur and the height of the pressure difference curve. The detailed results follow.

Type 1. Type 1 defects appeared in the bulbar septum superior to the crista supraventricularis immediately below the posterior cusp of the pulmonary valve. Only one patient fell into this category. The murmur was pansystolic with late systolic accentuation (Fig. 2). The right ventricular pressure was considerably raised $(63 / 7 \mathrm{~mm}$. $\mathrm{Hg})$. The pressure difference curve was rectangular in shape, showed an early maximum but no late systolic rise to parallel the intensity envelope of the murmur.

Types 2 and 3. Type 2 defects were located in the membranous septum at or near the commissure 


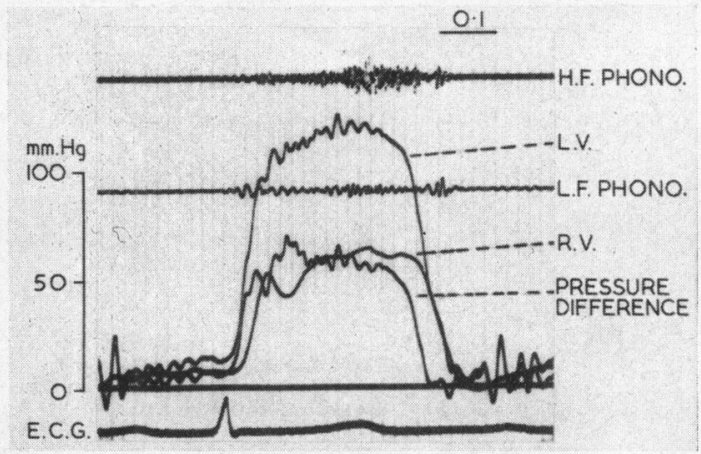

FIG. 2.-Simultaneous equisensitive pressure pulses in right and left ventricles together with pressure difference curve obtained by electronic subtraction, and phonocardiograms in a patient with Type 1 interventricular septal defect. Note that the murmur shows a late systolic accentuation which has no correlate in the pressure difference curve (H. F.-high frequency; L. F.-low frequency; paper speed-150 mm./sec.; time lines-0.02 sec.).

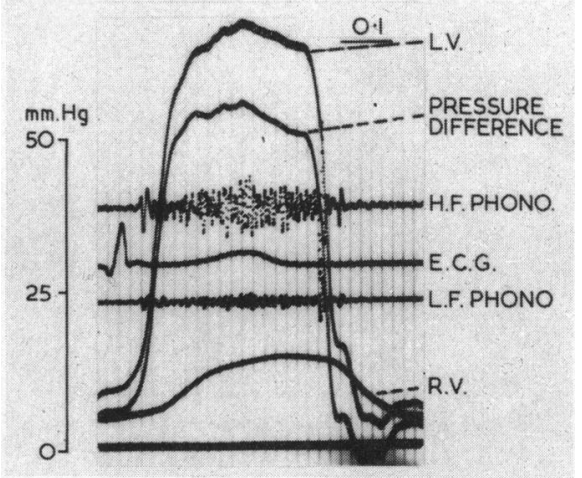

Fig. 3.-Recordings in a patient with Type 2 (membranous) interventricular septal defect. The right ventricular pressure pulse is damped. Note the rectangular shape of the pressure difference curve and the slight correlation with the intensity envelope of the murmur. The aortic component of the split second sound is buried in the murmur as observed in the high frequency recording.

between the anterior and right posterior aortic cusps. Type 3 defects appeared posteriorly and inferiorly to the Type 2 defects but were in the superior muscular septum. Though Type 2 is a membranous defect and Type 3 a muscular one, the two may in some cases be in such close proximity anatomically that only post-mortem examination can differentiate them. For this reason they are discussed together. Eleven cases fell into these two groups.

When the right ventricular pressure was normal or slightly raised the murmur was pansystolic and the shape varied from plateau to crescendo-decrescendo and late crescendo (Fig. 3, 4, and 5). The pressure difference curves were rectangular, paralleling closely the shape of the left ventricular pressure pulse. The difference curves rose abruptly reaching an early systolic peak which was maintained throughout systole, and fell off abruptly in late systole. Variations in the shape of the intensity envelope of the murmur in different cases were not accompanied by similar variations in the pressure difference curves.

In three patients with high right ventricular pressures and large shunts the murmurs were early

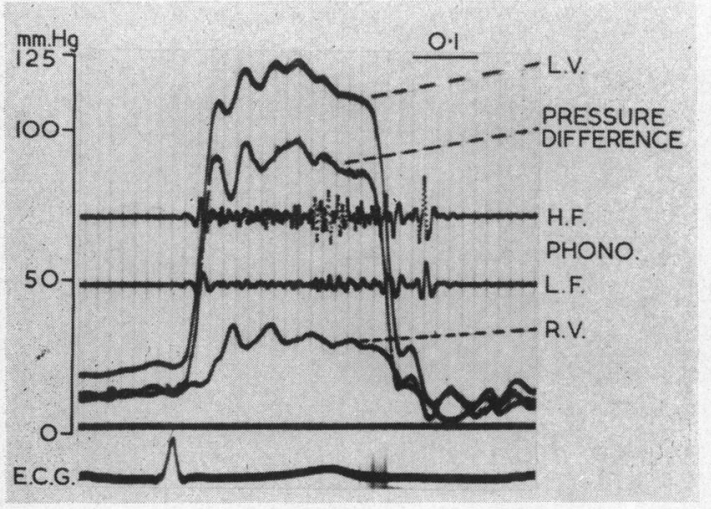

FIG. 4.-Another patient with a Type 2 (membranous) defect. Here, the murmur, though holosystolic, exhibits a late systolic accentuation which is not reflected in the pressure difference curve.

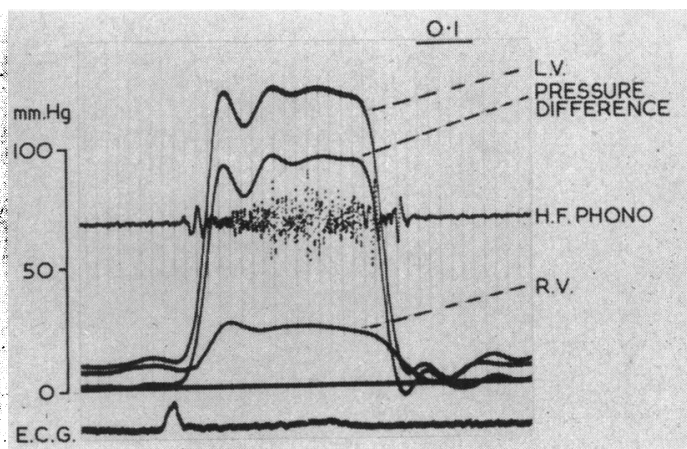

Fig. 5.-Also a Type 2 defect. Though the pressure difference reaches an early peak, the murmur is relatively faint during this phase of the cycle. 


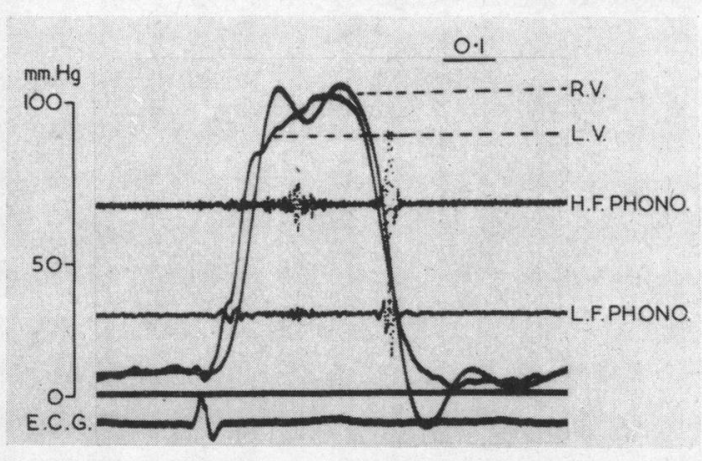

FIG. 6.-A high-pressure, high-flow interventricular septal defect exhibiting only a short mid-systolic murmur. The pressure pulses in both ventricles are almost identical, exhibiting small differences of doubtful significance. The highest intensity of the murmur occurs at a time when the pressures are equal. Note that the second sound is single.

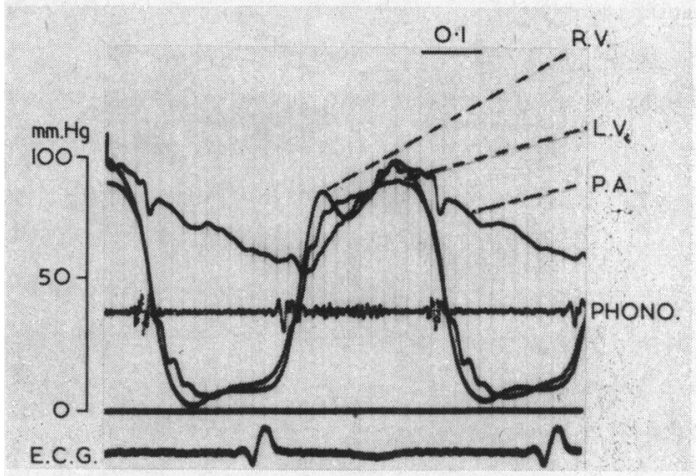

FIG. 7.-This case is similar to the previous one (Fig. 6). The murmur begins after the first sound and ends abruptly in mid-systole.

and mid-systolic or mid-systolic alone and of crescendo-decrescendo configuration (Fig. 6 and 7). All these patients had pressures in the right ventricle equal to systemic pressures. The pressure differences between the two ventricles were small and variable and probably of little hæmodynamic significance in view of possible pressure artefacts. For these reasons, the pressure difference curves were not recorded in these cases. There was no correspondence between the intensity envelope of the murmur and the apparent pressure difference.

Type 4. Type 4 defects were located in the muscular septum either in the inferior or outflow portion. These are the defects commonly referred to in the published reports as "muscular defects" (Fig. 8a and b). Five cases were encountered. The murmur began with the first sound and continued to mid-systole in decrescendo fashion. The pressure difference curve was again rectangular and virtually paralleled the left ventricular pulse reaching a maximum in early systole and continuing to the end of systole long after the murmur had disappeared (Fig. 8a). In one patient of this group phenylephrine hydrochloride $0.25 \mathrm{mg}$. was administered intravenously and caused a distinct rise in both left and right ventricular pressures. The left ventricular pressure rise was more pronounced and was accompanied by a distinct increase in the pressure differences. The murmur, however, remained unchanged (Fig. $8 \mathrm{~b}$ ).

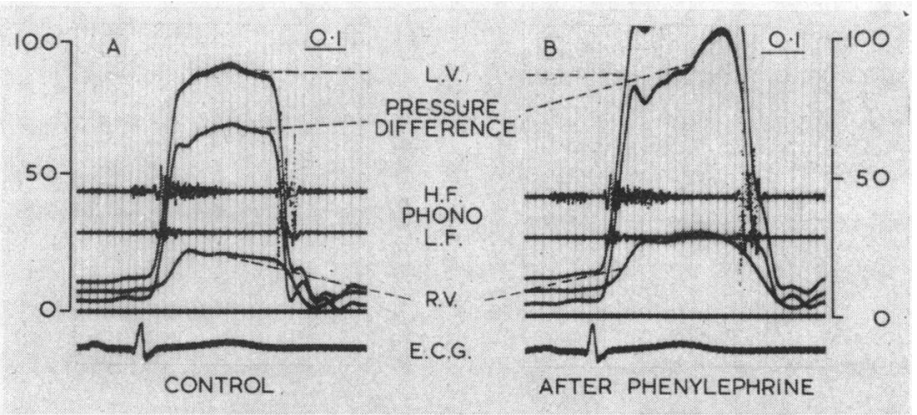

Fig. 8.-Type 4 (muscular) defect. In $\mathrm{A}$, the control tracing, the murmur is early and mid-systolic and decrescendo, ending at a time when the pressure difference is still at a maximum. In $B$, after intravenous phenylephrine, the pressures in both ventricles rise considerably, with a large increase in the pressure difference without a corresponding change in the duration or intensity of the murmur. 


\section{Discussion}

The murmur of interventricular septal defect has been variously described as being of plateau, crescendo, decrescendo, or late crescendo configuration (Bleifer, Donoso, and Grishman, 1960; Craige, 1960; Hollman et al., 1963; McKusick, 1958; Ongley et al., 1960). The duration of the murmur is usually pansystolic, extending beyond aortic valve closure, but under certain conditions may end at varying intervals before the second sound (Jefferson, Leatham, and Sloman, 1959; Leatham, 1958; Leatham and Segal, 1962; Vogelpoel et al., 1961). It is reported and generally accepted that a murmur that does not extend throughout systole is characteristically associated with small muscular defects or with large membranous defects that are accompanied by severe pulmonary and right ventricular hypertension (Leatham and Segal, 1962). Our own experience confirms these observations. In our group of five patients with small muscular defects (Type 4), a short murmur was indeed recorded. Similar murmurs were demonstrated in three patients with large defects (Types 2 and 3) associated with severe pulmonary and right ventricular hypertension.

Numerous authors have speculated about the mechanism of the short murmur in small muscular defects; it has been postulated that closure of the defect occurs with systolic contraction so that in late systole there is no, or virtually no, flow across the defect (Jefferson et al., 1959; McKusick, 1958). However, Vogelpoel et al. (1961) have presented evidence suggesting that the defect, in fact, remains patent throughout systole. They were able to intensify the murmur and make it pansystolic with the use of intravenous phenylephrine. These authors thought that the characteristics of the murmurs were dependent on the pressure gradient between the ventricles.

In our cases of small muscular defects, we were able to demonstrate a large pressure gradient between the ventricles that extended considerably beyond the "cut-off" point of the murmur as recorded on the phonocardiogram. We studied only one case of small muscular defect after the administration of phenylephrine, and found that the murmur did not lengthen or intensify despite the fact that the pressure gradient across the defect was clearly increased over the control (Fig. 8). Our single observation supports the hypothesis that the small muscular defect may indeed close in late systole while the pressure differential between the ventricles is still at a maximum. However, there is the possibility that the defect may remain open with flow changing from a turbulent to a laminar character, thus eliminating the murmur.

A short murmur has also been described in patients with ventricular septal defect and pulmonary hypertension and increased pulmonary resistance (Leatham and Segal, 1962; Lessof, 1959). The pressure pulse records in two patients of this series (Fig. 6, 7) who fall into this group do not suggest any hæmodynamic explanations for the time characteristics of the murmur. Though the right ventricular pressure exceeds that in the left ventricle during early systole, the maximum intensity of the murmur is slightly later in the cycle, at a time when the two pressures are equal. Furthermore, it is possible that apparent pressure differences in early systole may be artefactual.

While it has been shown that the time-intensity relations of the murmur of aortic and mitral stenosis vary directly with the pressure differences across the valves (Gordon et al., 1958; Gordon, Kirschner, and Moscovitz, 1961), we have not been able to demonstrate this relation in interventricular septal defect. This may be explained by differences in flow characteristics. In stenotic valvular lesions, blood flow is presumably unidirectional. In interventricular septal defect, however, the blood flow from each ventricle has two possible outlets which would lead to a complex flow interrelation between the ventricles and the great vessels. Such factors as velocity, turbulence, and inertia might well come into play, in addition to the pressure difference alone. One other possibility that should at least be mentioned is that a part of the murmur may be a phenomenon of the increased flow, and may actually originate in the pulmonary artery. In that case, no correlation at all would be expected with the pressure difference between the ventricles.

\section{SUMMARY AND CONCLUSIONS}

In 20 patients with isolated interventricular septal defects, correlation was attempted between the intensity envelope of the murmur and the continuous pressure difference across the defect. Simul- 
taneous equisensitive pressure pulses were recorded from both ventricles together with continuous pressure differences. Phonocardiograms were also recorded simultaneously. The defects varied in size and location and there were varying degrees of pulmonary hypertension.

In general, the shape of the continuous pressure difference was rectangular. The murmurs, however, varied widely from case to case in both duration and shape. Poor correspondence was observed between the time-intensity characteristics of the murmur and the pressure gradient across the defect.

It is concluded that the pressure relation between the two ventricles is not the only factor influencing the character of the murmur of uncomplicated interventricular septal defect.

The authors are indebted to Drs. Leslie A. Kuhn and Henry Mizgala, who also took part in this investigation.

\section{REFERENCES}

Baron, M. G., Wolf, B. S., Steinfeld, L., and Gordon, A. J. (1963). Left ventricular angiocardiography in the study of ventricular septal defects. Radiology, 81, 223.

Bleifer, S., Donoso, E., and Grishman, A. (1960). The auscultatory and phonocardiographic signs of ventricular septal defects. Amer. J. Cardiol., 5, 191.

Craige, E. (1960). Phonocardiography in interventricular septal defects. Amer. Heart J., 60, 51.

Gordon, A. J., Kirschner, P. A., and Moscovitz, H. L. (1961). Hemodynamics of Aortic and Mitral Valve Disease. Grune and Stratton, New York.

- Steinfeld, L., Dunst, M., and Kirschner, P. A. (1958). Correlation of heart sounds and murmurs with pressure during left heart catheterization. Circulation, 18, 979.

Hollman, A., Morgan, J. J., Goodwin, J. F., and Fields, H. (1963). Auscultatory and phonocardiographic findings in ventricular septal defect. A study of 93 surgically treated patients. Circulation, $28,94$.

Jefferson, K., Leatham, A., and Sloman, G. (1959). The diagnosis of small ventricular septal defects. In Proceedings of the British Cardiac Society. Brit. Heart J., 21, 580.

Leatham, A. (1958). Auscultation of the heart. Lancet, 2, 703, 757.

- , and Segal, B. (1962). Auscultatory and phonocardiographic signs of ventricular septal defect with left-to-right shunt. Circulation, 25, 318.

Lessof, M. (1959). Heart sounds and murmurs in ventricular septal defect. Guy's Hosp. Rep., $108,361$.

McKusick, V. A. (1958). Cardiovascular Sound in Health and Disease. Williams and Wilkins, Baltimore.

Ongley, P. A., Sprague, H. B., Rappaport, M. B., and Nadas, A. S. (1960). Heart Sounds and Murmurs. Grune and Stratton, New York.

Vogelpoel, L., Schrire, V., Beck, W., Nellen, M., and Swanepoel, A. (1961). The atypical systolic murmur of minute ventricular septal defect and its recognition by amyl nitrite and phenylephrine. Amer. Heart J., 62, 101 . 